\title{
SNRPN Upstream Reading Frame Protein
}

National Cancer Institute

\section{Source}

National Cancer Institute. SNRPN Upstream Reading Frame Protein. NCI Thesaurus.

Code C104856.

SNRPN upstream reading frame protein (71 aa, $\sim 8 \mathrm{kDa}$ ) is encoded by the human SNURF gene. This protein may be involved in the development of the brain. 